\title{
Beyond the Medium: Rethinking Information Literacy through Crowdsourced Analysis
}

\author{
Olga Boichak \\ Syracuse University \\ oboichak@syr.edu \\ Brian McKernan \\ Syracuse University \\ bmckerna@syr.edu \\ Benjamin A. Clegg \\ Colorado State University \\ Benjamin.Clegg@,colostate.edu \\ Nancy McCracken \\ Syracuse University \\ njmccrac@syr.edu \\ James Folkestad \\ Colorado State University \\ james.folkestad@colostate.edu
}

\author{
Jordan Canzonetta \\ Syracuse University \\ jncanzon@syr.edu \\ Sarah Taylor \\ taylmail59@gmail.com
}

Kate Kenski

University of Arizona

kkenski@email.arizona.edu

Carsten Østerlund

Syracuse University

costerlu@syr.edu

Jennifer Stromer-Galley

Syracuse University

jstromer@syr.edu

\author{
Niraj Sitaula \\ Syracuse University \\ nsitaula@syr.edu \\ Patrícia Rossini \\ Syracuse University \\ pgoncalv@syr.edu
}

Rosa Mikeal Martey

Colorado State University

rosa.martey@colostate.edu

\author{
Roc Myers \\ Pertis LLC \\ roc.myers@gmail.com
}

\begin{abstract}
Information literacy encompasses a range of information evaluation skills for the purpose of making judgments. In the context of crowdsourcing, divergent evaluation criteria might introduce bias into collective judgments. Recent experiments have shown that crowd estimates can be swayed by social influence. This might be an unanticipated effect of media literacy training: encouraging readers to critically evaluate information falls short when their judgment criteria are unclear and vary among social groups. In this exploratory study, we investigate the criteria used by crowd workers in reasoning through a task. We crowdsourced evaluation of a variety of information sources, identifying multiple factors that may affect individual's judgment, as well as the accuracy of aggregated crowd estimates. Using a multi-method approach, we identified relationships between individual information assessment practices and analytical outcomes in crowds, and propose two analytic criteria, relevance and credibility, to optimize collective judgment in complex analytical tasks.
\end{abstract}

\section{Introduction}

Information literacy scholars have been concerned with the ways the public garners and evaluates information in a variety of settings, such as in learning, and in news consumption as part of the work of citizenship. Information literacy scholarship concerns itself with people being aware and mindful of the meaning received from media sources [1]. To understand how people critically evaluate content in digital media outlets, from websites to blogs to social media, affects their ability to produce sound reasoning, we consider how people become aware of, evaluate, and interpret information from a variety of digital sources.

Information literacy skills are directly related to critical thinking, especially critical evaluation of information [2], [3]. Cognitive models of information literacy propose four components: knowledge structures relating the real world and the self, personal locus (individual information needs and abilities), experience, and competencies [4]. Applied to digital contexts, this model emphasizes the ability to think deeply about media experiences and become aware of 
media content, industries, and effects [5]. Although information literacy models vary widely, most integrate reader attitudes about the information in question with existing knowledge of the world for the purpose of making judgments or evaluations about the meaning of tasks or ideas [6]. An underlying assumption of these approaches is that information literacy leads to improved critical thinking, which, in turn, lets reasoners produce better analyses and makes them more effective in decision making.

Research also suggests that information literacy and the decision making that results from such is subject to processes of cognitive biases-shortcuts in thinking that are often beneficial [7], [8], but sometimes can lead to faulty reasoning [9]. For example, confirmation bias is the tendency to seek and positively evaluate information that aligns with one's hypotheses or opinions, and to discount information that runs contrary to such. In the context of information literacy, cognitive biases can be detrimental.

In recent years, a variety of platforms and websites have established the utility of crowdwork to help solve complex problems. Perhaps, the most widely adopted example is Wikipedia, in which anyone can create entries, edit them, and talk with others about the content. Crowdsourcing potentially holds promise in the evaluation of information to support decision making. For instance, after the Boston marathon bombing in 2013, members of the public sifted through hundreds of hours of video and thousands of pictures of the crowd to identify the attackers.

This paper presents an exploratory study that examines how individuals' critical assessment of information sources affects judgment of a complex problem. In doing so, we take up boyd's [10] call to interrogate information literacy as a concept by critically analyzing practices of consumption, evaluation, and interpretation of information on digital media. We collected and analyzed participants' assessments of information sources including Wikipedia, news articles, and social media accounts, in order to identify the relationships between individual source assessment practices and analytical outcomes. We ask: Do those who perform more effective evaluations of information sources generate better analyses in complex reasoning tasks? How can our findings be used to improve media literacy training? Finally, how can our findings inform ways to mitigate cognitive biases in crowds?

\section{Information Literacy and Crowds}

Influences of the digital information technology complicate traditional notions of information literacy, due particularly to changed practices in circulation, curation, and consumption of content [1], [4]. Information literacy now requires even greater skills, as messages are circulated and embedded in different, unfamiliar forms and genres of digital media, and contextualized through sharing practices by social tie networks - a process which activates cognitive biases that tend to short-circuit our critical facilities [11]. Reasoning and analytical skills are thus vital to information literacy - a recent topic of international discussion in the wake of the spread of "fake news" and its impact on political campaigns and elections. As false or misleading digital news content proliferates, consumers of digital media, scholars, and citizens alike are asking how to develop literacies to critically engage with digital media content.

Hawisher and Selfe [12] suggest orientations to information literacy (and the "acquisition" of it) are related to individuals' cultural ecologies and must be acknowledged in discussions about these literacies. Recent work in information literacy focuses on the set of skills required for an individual to critically evaluate information in online environments, such as identifying advertising in search results or social media, recognizing sponsored content in blogs, or assessing news sources for accuracy and credibility.

Information literacy training prompts readers to examine dynamic media contexts as new areas of learning based on existing schemas of information [13], [14]. Thus, information literacy skills help people interpret mediated messages and filter them through developed knowledge structures, such as personal experience [4]. People often tend to align their preexisting biases with their perspectives on media resources [15]. People with high information literacy, however, can make better decisions about, and can better construct meaning from, the information they consume [4].

Recently, researchers and the United States federal government have explored ways to capitalize on crowd-based work to support more complex reasoning tasks. Several citizen-science projects exist, such as Zooniverse, which allows researchers to harness the crowd in a variety of identification tasks, such as examining photos to determine what types of leaves are in the images. An MIT research group created EyeWire, which is a citizen-science project to map neural networks. Recently, U.S. national intelligence agencies have explored ways to leverage the idea of crowdwork within the intelligence community to conduct complex reasoning tasks, via the CREATE program [16].

When it comes to crowds, information literacy plays an important, if at times detrimental, role. When asked to assign a value to an object in question, crowd 
estimates are often more accurate compared to individual estimates due to the wisdom of the crowd effect - a statistical phenomenon which purports that errors in individual reasoning cancel each other out in aggregate measures [17]. Thus, while individuals might be prone to cognitive biases that can negatively affect reasoning, aggregates of individuals' estimates should ideally converge around the ground truth. Yet, wisdom of crowds is not without flaws. Studies have shown that crowd estimates can be easily swayed by social influence: encouraging individuals to revise or reconsider their opinions inadvertently introduces bias in statistical aggregates [17]. This paradox helps illuminate unanticipated effects of contemporary information literacy training: encouraging readers to critically evaluate information falls short when the judgment criteria underlying information literacy are unclear; reader opinion on what constitutes relevant and/or credible information might differ significantly across social groups and cultural contexts. In this study, we demonstrate how these discrepancies in information assessment criteria (caused by social influence) have the potential to introduce bias in collective judgment, and suggest ways to remedy the situation.

If the tasks that leverage the crowds are going to include complex reasoning problems that require critical evaluation of source information, research is needed to understand how individuals engage in source evaluation, and to consider ways to design crowd-tasks that effectively capitalize on crowdwork while mitigating challenges, such as social influence, cultural, or cognitive biases. Thus, this work provides a preliminary exploration of criteria people use to evaluate and interpret information from sources. We use original data from a larger study on crowd-sourced reasoning to suggest that manipulating individual opportunity to assess information allows us to improve analytical reasoning and decision making by individuals and potentially, in the future, by crowds. Based on our findings, we develop a series of recommendations on improving information literacy training, especially in the context of crowd-sourced analytical tasks.

\section{Relevance and Credibility as Measures of Information Assessment}

Relevance and credibility were established as measures of information assessment because they are criteria that indicate critical engagement with resources and their content. Studies in the field of Rhetoric and Composition outline detailed methods for evaluating the credibility and relevance of a source in college research writing courses, and prioritize these criteria for sound research practices when determining how to write a well-informed and researched report [18]-[20]. From these criteria for evaluation, we extract a set of conceptual ideals for critically engaging with resources in digital media.

Determining the credibility, relevance, and usefulness of a resource are precursors to critical analysis, which requires readers to synthesize information from resources and formulate an argument or hypothesis about the linkages between them [18]. Critical engagement through analyses of credibility and relevance moves readers beyond a mere summary of isolated resources and puts them into conversation with each other. Doing so demonstrates a mature analytical capacity to identify assumptions, detect clues and strategize about how to make sense of connections between related pieces of information.

Assessing a source's credibility is related to a reader's ability to consider how various accounts of an event align, misalign, verify, or disconfirm the ground truth of a particular occasion. For instance, if three resources contain a corroborated fact and one contains information contrary to the majority, this resource should be considered possibly untrustworthy or not credible. The same is true of the reverse; if a resource is perceived as a non-credible resource, but contains information that aligns with other credible resources, readers' ability to read around the erroneous information indicates strong critical analysis skills. Readers' evaluation of each source's credibility, if assessed well, will lead to a strong analysis and interpretation of how all sources in a given case work together to support a final argument or claim [20].

Rating a source's relevance implies readers are developing critical and analytical strategies for piecing together an answer to the case. Sifting through misleading, irrelevant, or tangential information requires organizational processes that build critical engagement with source materials. Selecting and prioritizing the most relevant information from a case shows readers' rhetorical choices in action. Discarding useless information indicates rhetorical skills because readers are building a case for their arguments, and want to choose the most persuasive information available to convince their audiences of the veracity of their judgments. Determining a source's relevance indicates readers have chosen to include specific information that bolsters their arguments and strengthens their ethos, which suggests rhetorical acumen and critical reasoning aptitude. Thus, these criteria were selected for measuring readers' levels of engagement with sources. 


\section{Data and Methods}

This study combines quantitative and qualitative analyses to assess the impact of criteria employed by people when evaluating documents on the quality of their analytic outcomes. In the study, 88 participants from the United States were recruited using Amazon Mechanical Turk (MTurk). The participants had a bachelor's degree or equivalent. There were no cultural, material, or socio-economic criteria for the workers, helping us gain a range of experiences and knowledge around information literacy.

Participants were asked to analyze information and write a short report ( $300-500$ words) addressing a complex problem with a known answer: why a perpetrator might have attacked his victim. The problem, 'Unusual Suspects', was written by the authors using the structural elements of a historical case that took place in Ukraine in 2014: a young athlete infamously assaulted two journalists covering an anti-government demonstration. For their analyses, participants were instructed to use only six documents created for the study: fictional but realistic news media reports (one coming from a watchdog organization called 'Human Rights Watch', another one from the Washington Post), social media posts (the perpetrator's alleged Facebook account), a Wikipedia article, and two tabloid articles. The documents were deliberately created to include relevant and irrelevant, as well as credible and non-credible information and various genres of communication.

Participants performed their analyses using an online tool created by the researchers that provided a template for the report and an interface to view and rate each source for relevance and credibility using a system of 1 to 5 stars. After reading the source materials, they were asked to write a report in the online tool that explained their reasoning, justifications, (including their ratings of documents), assumptions, and final conclusions regarding the case in question. The document ratings and reports were then analyzed quantitatively, and the explanations were subjected to an inductive qualitative analysis, to identify the criteria the readers used to determine which information should be used to answer the question they were asked.

The codebook for systematic analysis established categories for assessing participants' quality of reasoning with a set of 15 codes, developed through eleven iterative stages of coder testing, debriefing, and revision. Codes were developed and revised with the aim of evaluating how well participants could reason through a complex problem. Codes that related to reasoning evaluated participants' writing and analysis of the problem, specifically in areas related to assumptions, quality of reasoning, correct answers, gaps in information from the resources, and evaluation of the credibility and relevance of the resources. Criteria for each code were determined by qualities such as accuracy, presence or absence, and type of explanation. In teams of two, coders independently coded sets of participants' reports and then adjudicated discrepancies with their coding partners.

\section{Findings and analysis}

The findings of the study consist of four parts and focus on illuminating discrepancies in information literacy patterns among two groups of participants $(n=88)$ : those who arrived at an incorrect or a somewhat correct solution to a complex analytical problem $(\mathrm{n}=48)$, and those whose judgments were marked as correct $(n=40)$. First, we analyze inter-group differences in distributions of crowdsourced ratings of relevance and credibility of various media sources (5.1); second, we analyze pairwise correlations between ratings of relevance and credibility and compare them between groups (5.2); third, we qualitatively examine the participants' explanations on why they found certain sources and/or pieces of information worthy of consideration (5.3); and finally, we use regression analysis to measure the effects of correct evaluations of relevance and credibility of sources on analytic outcomes (5.4). We conclude with a series of recommendations on using the concept of information literacy to improve reasoning outcomes in crowdsourcing systems.

\subsection{Crowdsourced assessments of credibility and relevance across media sources}

Information literacies are often shaped by social contexts, cultural values, educational practices, political views, and economic trends [13], all of them social influences that affect how people make sense of information through media. Comparing among two groups of participants: those who came to an incorrect or a somewhat correct judgment, and those whose judgment was marked as correct, we observed significant differences in rankings of credibility and relevance of the media sources: a U.S.-based news media outlet (resembling The Washington Post), two tabloids (resembling a British Daily Mail and a Ukrainian tabloid, Tabloid.ua), a social media account (resembling Facebook), a free-content encyclopedia (resembling Wikipedia), and a human rights watchdog organization's website.

Analyzing the discrepancies among the two groups, we observed differences between credibility ratings in 
three media sources: the Washington Post, Daily Mail, and Wikipedia (Figure 1). Readers who, on average, distrusted and/or dismissed crucial information from the Washington Post as not credible, were more likely to produce poor analytic outcomes. This finding could be explained by the various cultural contexts of information consumption - certain social groups tend to distrust sources with an alleged socially liberal bias, such as the Washington Post, which might have caused some readers to automatically dismiss key information from this source, producing an incorrect judgment. We also observed that, although these differences were not statistically significant, readers who got the judgment incorrect were more likely to trust the Daily Mail, a British tabloid, while at the same time distrusting Wikipedia, a crowdsourced encyclopedia, despite the latter providing key information corroborating an argument for a correct judgment.

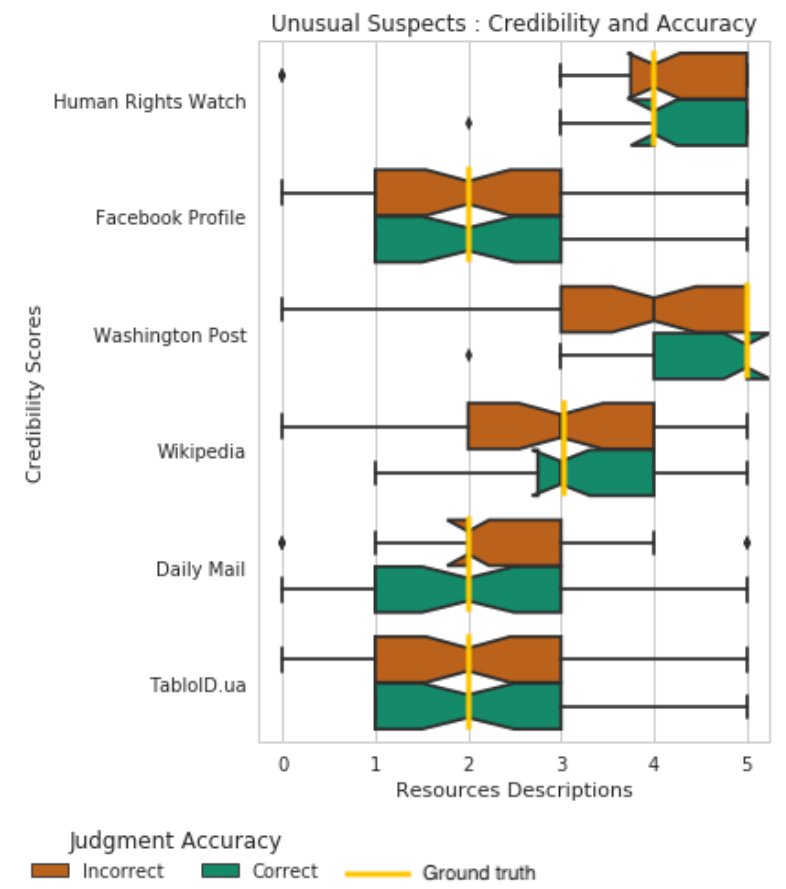

Figure 1. Crowdsourced assessments of credibility across various media sources.

We found differences between crowd ratings of relevance across all six media sources (Figure 2). Analysis demonstrates that readers who arrived at an incorrect judgment were also likely to misjudge the relevance of key information, regardless of the reputation of the medium. These readers based their reasoning on an article from a watchdog organization with little relevant content, and placed disproportionate trust in the information from the perpetrator's Facebook account and information from a tabloid, while dismissing the highly relevant information from the Washington Post and Wikipedia.

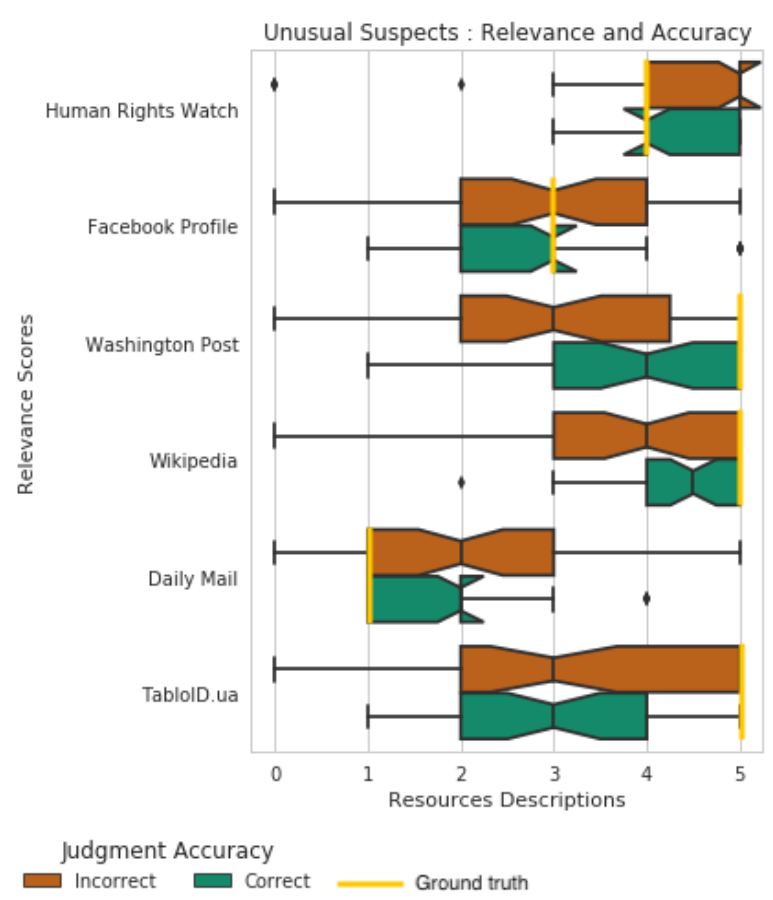

Figure 2. Crowdsourced assessments of relevance across various media sources.

These findings point to poor judgment in determining which information is relevant among reasoners who got the judgment incorrect: incidentally, the documents from the Washington Post and Wikipedia did not contain the perpetrator's name, but provided useful information on the political context in which the incident took place, explaining the key actors and interests involved. This could be explained by a possible lack of critical awareness skills among the participants who got the judgment wrong: these skills that are crucial for interrogating sources like Facebook, wherein readers should not take content at face value. Additionally, readers may isolate particular pieces of information that are easily processed, which may cause them to fail to see how several sources work together to solve a complex question. Finally, participants who reached incorrect conclusions might have been prone to confirmation bias [21], wherein they would use their reasoning skills selectively, dismissing information that was inconsistent with their political beliefs. Compared to the reasoners whose judgment was correct, we observed that reasoners from the incorrect category had a difficulty assessing both relevance, and credibility, which subsequently hindered their analysis. 


\subsection{Spearman's Rank Correlation between relevance and credibility across media sources}

Given that reasoners who get the judgment incorrect on average tend to misidentify both relevance and credibility of key information, we used Spearman's rank correlation to analyze pairwise statistical relationships between crowdsourced ratings of relevance and credibility in each group (those who got the judgment incorrect and correct), to see how both of these criteria might work together in contributing to information literacy.

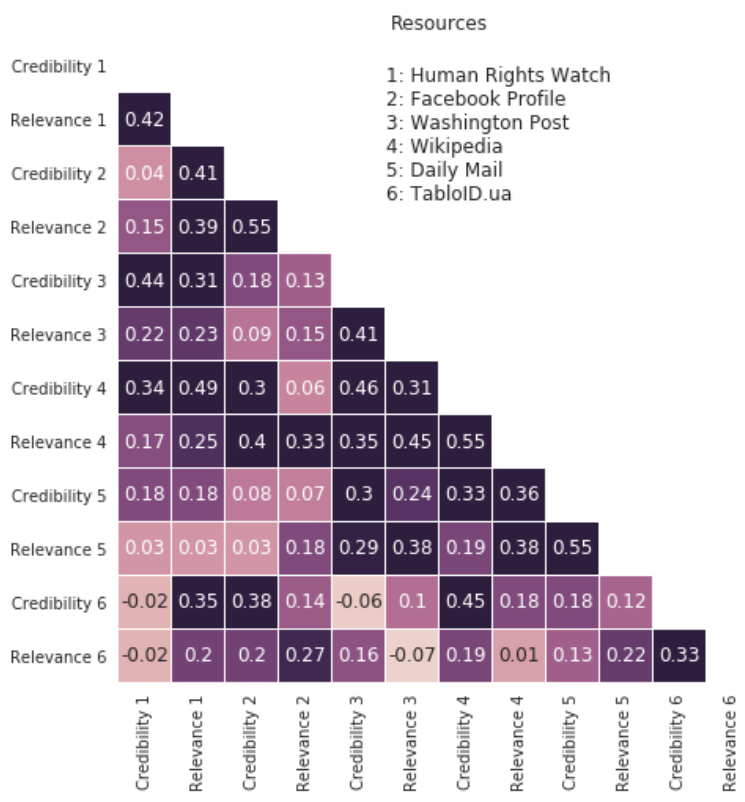

Figure 3. Spearman's Rank Correlation between relevance and credibility ratings across media sources (Incorrect judgment, $n=48$ )

This analysis suggests statistically significant pairwise correlations between relevance and credibility for each source among users who got the judgment incorrect (Figure 3). The average statistically significant pairwise correlation among all sources for this group was 0.46 .

Reasoners who got the judgment correct (Figure 4) seemed to be better able to distinguish between the two criteria: pairwise correlations in this group were much lower, and few were statistically significant. For comparison, the average correlation among all sources for this group was 0.22 , although only two pairwise relevance/credibility correlations - that for Human Rights Watch (source 1, 0.34), and that for TabloID.ua (source $6,0.46$ ) - were statistically significant.

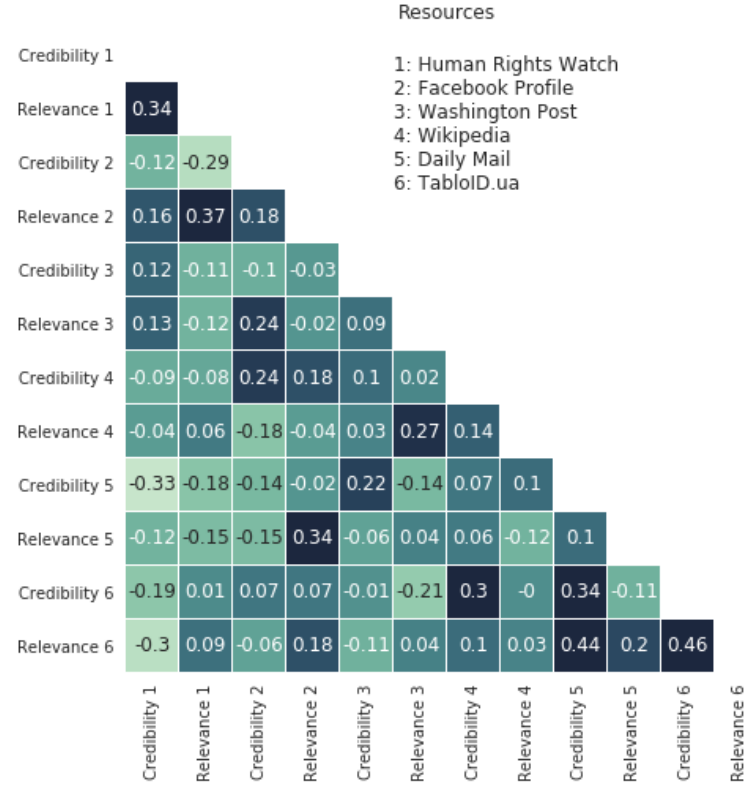

Figure 4. Spearman's Rank Correlation between relevance and credibility ratings across media sources (Correct judgment, $n=40$ )

Having observed a set of patterns that lead to incorrect judgment outcomes in a group of participants, we identified problematic information assessment behaviors, in an attempt to establish a set of thresholds that predict poor analytical outcomes based on the ratings of relevance and credibility.

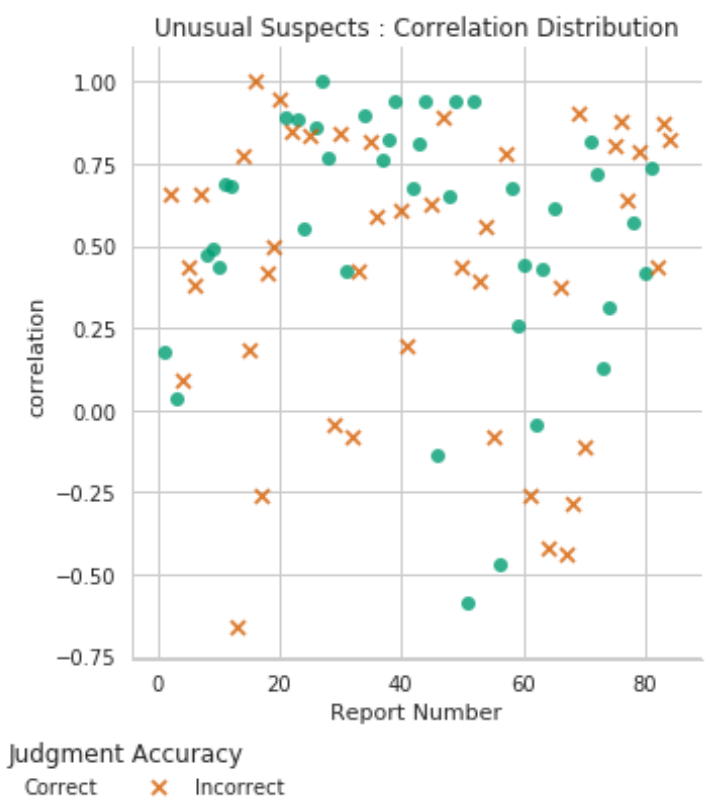

Figure 5. Spearman's Rank Correlation between relevance and credibility ratings for each participant ( $n=84$, as 4 participants in the study did not provide any ratings) 
We hypothesized that participants who confuse relevance and credibility would have a high Spearman's rank correlation among these two ratings for all six sources, which would then allow to eliminate their estimates from consideration, to improve crowd outcomes. Figure 5 does not confirm this assumption: removing participants with high correlation among ratings of relevance and credibility yields only marginal improvement in crowd accuracy. This suggests there is no simple systematic confounding of credibility and relevance within all sources driving worse performance in crowds.

Conclusively, findings in this subsection suggest that reasoners who are better able to distinguish between the categories of relevance and credibility, which we infer suggests higher information literacy skills, are better able to discern the critical information to help them arrive at the best judgment. In the next subsection, we further examine this finding by conducting a qualitative analysis of participants' explanations of their information assessment.

\subsection{Analyzing participants' explanations of information assessment}

For this analysis, we examined the readers' comments on why they deemed certain sources and/or pieces of information worthy of consideration. When readers articulated criteria for including/excluding certain information in their judgment, these criteria were recorded in a frequency table. Unsurprisingly, the most frequent criteria used by the readers to explain their choices were credibility (35 out of 88 ), and relevance ( 21 out of 88 ), as these were the two options suggested by the tool. While there is a significant overlap between the criteria that readers use to distinguish between good and bad media sources, 10\% of readers from both groups tend to evaluate information based on credibility of sources (16 out of 40).

Table 1. Inductive evaluation of readers' explanations

\begin{tabular}{l|ccc} 
& $\begin{array}{c}\text { Total } \\
(88)\end{array}$ & $\begin{array}{c}\text { Incorrect } \\
(48)\end{array}$ & $\begin{array}{c}\text { Correct } \\
(40)\end{array}$ \\
\hline $\begin{array}{l}\text { At least one } \\
\text { criterion }\end{array}$ & 67 & 39 & 28 \\
\hline $\begin{array}{l}\text { Two or more } \\
\text { criteria }\end{array}$ & 18 & 12 & 6 \\
\hline Credibility & 35 & $\mathbf{1 9}$ & 16 \\
\hline Relevance & 21 & $\mathbf{1 4}$ & 7
\end{tabular}

Consistent with our other findings (see section 5.1), participants who got the judgment incorrect disproportionately prioritized relevance of information in their judgment (14 out of 48). In some instances, readers also confused relevance with reliability, corroborating the findings from section 5.2. As one participant explained, "The Washington post seemed to be reliable as it explained the groups involved in the protests". This identification of the key information in the source is actually about relevance, not credibility. While source credibility plays an important role in arriving at a correct judgment, a disproportionate focus on information relevance may in fact lead readers astray, making them more likely to fall for cognitive biases. In the next section, we measure the effects of crowd's ratings of relevance and credibility on the outcome of reasoning.

\subsection{The effects of correct evaluations of relevance and credibility on analytical outcomes}

We conducted an ordinary least squares (OLS) regression to consider the difference between the crowd ratings of relevance and credibility, and the "ground truth" rating provided by the team of authors of the materials. The calculations have been performed in $\mathrm{R}$ statistical software [22]; after the results have been obtained, we ran the Variance Inflation Factor to test for multicollinearity (none detected). We also ensured our model satisfied regression assumptions by running GVLMA, a global validation of linear model assumptions (all assumptions satisfied).

Table 2. OLS regression estimates

Variables Coef. Estimate

\begin{tabular}{ll}
\hline Intercept & $1.785(0.258)^{* * *}$ \\
\hline Human Rights Watch_credibility & $-0.048(0.12)$ \\
\hline Human Right Watch_relevance & $0.008(0.132)$ \\
\hline Facebook_credibility & $-0.203(0.088)^{* *}$ \\
\hline Facebook_relevance & $0.095(0.085)$ \\
\hline Washington Post_credibility & $0.171(0.102)^{*}$ \\
\hline Washington Post relevance & $0.145(0.074)^{*}$ \\
\hline Wikipedia_credibility & $-0.125(0.097)$ \\
\hline Wikipedia_relevance & $0.219(0.102)^{* *}$ \\
\hline Daily Mail_credibility & $-0.130(0.083)$ \\
\hline Daily Mail_relevance & $-0.151(0.086)^{*}$ \\
\hline TabloID.ua_credibility & $0.180(0.084)^{* *}$ \\
\hline TabloID.ua_relevance & $-0.063(0.07)$ \\
\hline Model Performance & 88 \\
Observations & 0.3198 \\
\hline R-squared & 0.2109 \\
\hline Adjusted R-squared & 2.94 \\
\hline F(12,75) & p-value: 0.002
\end{tabular}

Note: Standard errors in parentheses. $* p<0.1, * *$ $p<0.05, * * * p<0.01$ 
The findings suggest a significant inverse relationship between credibility of information from Facebook and judgment accuracy - the more credible a reasoner rated the resource, the less likely they were to arrive at a correct judgment. There is also a positive relationship between relevance and credibility of information from the Washington Post; reasoners who give high ratings to this source are more likely to arrive at a correct judgment. Moreover, understanding the relevance of information from Wikipedia increases the likelihood they arrive at a correct conclusion. However, finding relevant information in a tabloid makes one less likely to answer the question correctly, while believing a first-person account from a different tabloid raises the likelihood of one's judgment being correct. As this analysis suggests, information literacy skills go beyond the medium through which information is presented. To be successful in solving the problem from the case, readers needed to fully consider the content in each resource, regardless of their a priori perceptions of the resource's reputation.

\section{Discussion}

Based on the analysis performed in the previous section, we draw the following conclusions:

1. Reasoners with poor information literacy skills have a difficulty assessing relevance and credibility of information, which subsequently hinders their analysis.

2. Reasoners who are better able to distinguish between the categories of relevance and credibility, which we infer suggests higher information literacy skills, are better able to discern the critical information to help them arrive at the best judgment.

3. While source credibility plays an important role in arriving at a correct judgment, a disproportionate focus on information relevance may be more likely to activate cognitive biases.

4. Information literacy skills go beyond the relevance and credibility of the medium through which information is presented - a disproportionate focus on the medium's credibility makes reasoners disregard potentially useful information, which hinders their analysis.

According to boyd [11], the concept of information literacy should encompass the ways in which cognitive biases and cultural contexts influence information consumption, evaluation, and interpretation, both among individuals, as well as in crowds. For instance, in a widespread belief that Wikipedia is "unreliable" as it is comprised of crowdsourced contributions [11], we have observed readers placing more trust in questionable sources such as tabloids, prioritizing relevance of information over its credibility. This leads to erroneous judgments by making participants more likely to include irrelevant or deliberately misleading content in their reasoning.

Participants may also evaluate information based solely on the medium, ignoring the content of the message. A possible explanation of this behavior is the practice to employ a two-step process in information assessment: first, by dismissing information an individual does not find credible (based on the media source), and then deciding upon the relevance of the "acceptable" media sources. Consequently, participants with less developed media literacy skills might not view information as relevant unless they believe it is credible - an explanation which highlights the blurred line between relevance and credibility, and leads to the high correlations among their rankings of relevance and credibility (see section 5.2). Based on such observations, a thorough understanding of reader process and judgment criteria underlying information literacy is crucial for mitigating biases in collective judgment.

The findings demonstrate that participants who perform more effective evaluations of information sources indeed produce better analytic outcomes. This highlights the importance of information literacy in digital settings, and how criteria for credibility and relevance can be used to improve analytical outcomes in complex crowdsourced tasks. Following Vraga, Tully, and Rojas's work [16], we reaffirm the importance of developing critical information literacy awareness. They assert,

To make the informed decisions that are key for democratic participation, people need to evaluate the merit of news messages critically and accurately. Understanding how hostile interpretations can be minimized is crucial so that the news media can facilitate and encourage rational decision making.

Results from this study highlight potential areas for media literacy training, which is often uneven and varies widely across different cultural ecologies [12].

There are a few known limitations to this study. Firstly, some of the sources the participants were asked to evaluate were created to look like non-U.S.-based media in English language. While the alleged sources of the information were clearly indicated and labeled (for instance, "an excerpt from a tabloid", "a Wikipedia article"), it is possible that some U.S.-based readers might have been wary about making definitive statements about a foreign source they were unfamiliar with. Secondly, in an early version of this experiment, most documents had not been formatted to resemble 
the original sources - the documents were introduced as a tabloid, a Washington Post article, etc., but were not made to look like the original sources, aside from an alleged Facebook page. We do not know whether formatting influenced the readers' understanding of the content; however, to mitigate this uncertainty in the future experiments, the documents have since been formatted in close likeness to the media sources they purport to be. Future studies will evaluate the effect of formatting on readers' evaluation of information presented in the sources.

\section{Conclusions and Implications for Crowdsourcing Performance}

The implications of our exploratory analysis suggest that participants tend to inappropriately weight relevance and credibility, often conflating the two concepts. Moreover, an overreliance on relevance or credibility category alone when assessing source materials tends to lead to participants to arriving at the wrong judgment. Crowdsourcing source evaluation has the potential to help clarify and improve evaluation of information from different source materials. In our study, some of our participants do seem to understand the two concepts and apply them accurately to our sources. In the context of a crowd-enabled source evaluation platform, perhaps those more adept reasoners could help those who are less clear on the concepts to better understand what the two concepts mean.

These findings also have a series of implications for improving information literacy skills to optimize crowdsourced outcomes in complex analytical tasks. In this context, information literacy could be used to improve individual reasoning outcomes in crowdsourcing systems in two ways:

Training: by providing the participants with information literacy training that focuses on the distinction between relevance and credibility, and the degree to which both relevance and credibility can be partial.

Nudges: if users repeatedly rank different sources of information with similar relevance and credibility, the system could alert them of this possible confusion. High pairwise correlations between relevance and credibility could also serve as a flag to down-weight or even dismiss a user's contributions in crowdsourcing systems.

Our findings contribute to understanding of media literacy by illuminating the impact of individual abilities to think deeply about digital media experiences and their awareness about media content, industries, and effects. Establishing well-articulated criteria of relevance and credibility, discerning between the two, and looking beyond the medium on which information is presented are critical not only to information literacy skills, but to reasoning and ability to make well-informed judgments.

\section{Acknowledgements}

This research was supported by the Intelligence Advanced Research Projects Activity (IARPA) via the Department of Interior National Business Center contract number 2017-16121900004. The U.S. Government is authorized to reproduce and distribute reprints for Government purposes notwithstanding any copyright annotation thereon.

Disclaimer: The views and conclusions expressed herein are those of the authors and should not be interpreted as necessarily representing the official policies or endorsements, either expressed or implied, of IARPA, DoI/NBC, or the U.S. Government.

The authors thank SRC Inc. and its director Debi Plochocki for collaboration on creating TRACE, the analysis software used to collect data for this study.

\section{References}

[1] K. N. Rosenfeld, "Terms of the Digital Age: Realities and Cultural Paradigms," Handbook of Research on Media Literacy in the Digital Age, pp. 115-144, 2016.

[2] E. T. Arke and B. A. Primack, "Quantifying media literacy: development, reliability, and validity of a new measure," EMI Educ Media Int, vol. 46, no. 1, pp. 53-65, Mar. 2009.

[3] M. Feuerstein, "Media Literacy in Support of Critical Thinking," Journal of Educational Media, vol. 24, no. 1, pp. 43-54, Mar. 1999.

[4] W. J. Potter, Media Literacy. SAGE Publications, 2013.

[5] A. Maksl, S. Craft, S. Ashley, and D. Miller, "The Usefulness of a News Media Literacy Measure in Evaluating a News Literacy Curriculum," Journalism \& Mass Communication Educator, vol. 72, no. 2, pp. 228-241, Jun. 2017.

[6] D. F. Halpern, Thought and Knowledge: An Introduction to Critical Thinking, 5 edition. New York: Psychology Press, 2013.

[7] G. Gigerenzer and W. Gaissmaier, "Heuristic decision making," Annual review of psychology, vol. 62, pp. 451-482, 2011.

[8] D. G. Goldstein and G. Gigerenzer, "Models of ecological rationality: the recognition heuristic.," Psychological review, vol. 109, no. 1, p. 75, 2002.

[9] D. Kahneman and A. Tversky, "On the psychology of prediction," Psychological Review, vol. 80, no. 4, pp. 237-251, 1973.

[10] danah boyd, "Did Media Literacy Backfire?," Data \& Society: Points, 05-Jan-2017. [Online]. Available: 
https://points.datasociety.net/did-media-literacybackfire-7418c084d88d. [Accessed: 31-May-2018].

[11] E. Bakshy, I. Rosenn, C. Marlow, and L. Adamic, "The Role of Social Networks in Information Diffusion," in Proceedings of the 21st International Conference on World Wide Web, New York, NY, USA, 2012, pp. 519-528.

[12] G. E. Hawisher, C. L. Selfe, B. Moraski, and M. Pearson, "Becoming Literate in the Information Age: Cultural Ecologies and the Literacies of Technology," College Composition and Communication, vol. 55, no. 4, pp. 642-692, 2004.

[13] D. C. Phillips and J. F. Soltis, Perspectives on Learning. Teachers College Press, 2004.

[14] K. Tyner, Media Literacy: New Agendas in Communication. Routledge, 2009.

[15] E. K. Vraga, M. Tully, and H. Rojas, "Media Literacy Training Reduces Perception of Bias,” 2009.

[16] "Crowdsourcing Evidence, Argumentation, Thinking and Evaluation (CREATE).” [Online]. Available: https://www.iarpa.gov/index.php/researchprograms/create. [Accessed: 21-Sep-2018].

[17] J. Lorenz, H. Rauhut, F. Schweitzer, and D. Helbing, "How social influence can undermine the wisdom of crowd effect," PNAS, vol. 108, no. 22, pp. 9020-9025, May 2011.

[18] S. Chodorow, Writing a Successful Research Paper: A Simple Approach. Hackett Publishing, 2011.

[19] G. Harvey, Writing with Sources: A Guide for Students. Hackett Publishing, 2017.

[20] E. Losh, J. Alexander, K. Cannon, and Z. Cannon, Understanding Rhetoric: A Graphic Guide to Writing. Macmillan Higher Education, 2017.

[21] D. M. Kahan, E. Peters, E. Dawson, and P. Slovic, "Motivated Numeracy and Enlightened SelfGovernment," Social Science Research Network, Rochester, NY, SSRN Scholarly Paper ID 2319992, Sep. 2013.

[22] R Core Team, $R$ : A language and environment for statistical computing. Vienna, Austria: R Foundation for Statistical Computing, 2014. 